\title{
Le Champ-de-l'Air, premier hôpital psychiatrique du Canton de Vaud
}

\author{
Par Christian Müller
}

On peut certainement dire que le type de soins qu'une société donne aux personnes psychiquement souffrantes indique un certain état d'esprit collectif, est en quelque sorte un indice, un baromètre du niveau de culture. L'historien observe donc avec fascination cette période dramatique du début du siècle passé durant laquelle sont nés les premiers «asiles d'aliénés», distillations de l'esprit du temps nouveau. Il y a quelques années encore, personne n'aurait osé émettre des doutes quant à l'origine de ces premiers hôpitaux psychiatriques en Europe. D'un accord tacite, on soutenait l'hypothèse que les motifs principaux qui conduisirent les dirigeants de cette époque lointaine à la construction de ces établissements furent des principes humanitaires, le réveil d'une conscience sociale, la responsabilité morale du sort de ces infortunés. L'hôpital psychiatrique fut considéré comme l'expression d'une volonté philanthropique soutenue par l'avènement de la médecine et ses principes de causalité physiologique incluant de plus en plus le domaine de la maladie mentale.

Mais notre temps voit apparaître des opinions différentes. Ainsi, tout d'abord Foucault en France et plus tard Dörner en Allemagne, se basant sur des documents contemporains de l'époque, émettent l'opinion que le motif sous-jacent principal de la construction des hôpitaux psychiatriques fut d'ordre économique. Dörner va même jusqu'à prétendre que la société bourgeoise naissante, enrichie par l'industrialisation poussée, sentait le besoin d'écarter et de mettre à l'ombre les sujets non productifs en les enfermant dans des asiles d'aliénés.

Autre fait nouveau et troublant: il y a deux cents ans, partout en Europe, les malades mentaux se trouvaient dans les annexes insalubres des hospices généraux, à Lausanne à la Mercerie. La séparation des malades mentaux des malades physiques et donc la construction d'asiles pour aliénés, fut saluée comme un progrès important. Aujourd'hui on est en train de faire marche arrière. N'assistonsnous pas à l'adoption d'une loi révolutionnaire en Italie qui, non seulement interdit la construction d'hôpitaux psychiatriques, mais qui ordonne la fermeture des hôpitaux existants et le retour des malades psychiquement atteints dans les services de chirurgie, de médecine interne, etc.

Ces réflexions m'ont amené à jeter un coup d'œil sur l'histoire du premier hôpital psychiatrique vaudois, le Champ-de-l'Air. M. Claude Secrétan, dans son 
excellent livre sur l'Hôpital de Cery, a déjà consacré un important chapitre au sujet qui nous intéresse.

Revenons aux origines. Le jeune Etat de Vaud rachète en 1806 le bâtiment de la Cité-Dessous, un hôpital jusqu'alors propriété de la ville de Lausanne. Quelques aliénés y vivent dans des conditions misérables. De même que le GrandConseil envisage, à partir de 1809, la construction d'un hospice cantonal, il projette aussi la création d'une maison d'aliénés. Cela ne va pas de soi. On en parle souvent dans les séances du Grand-Conseil entre 1809 et 1810, et le projet va revenir encore plusieurs fois devant le Parlement. Finalement, une décision est prise sur la base d'un rapport de la Commission nommée pour l'examen d'un projet de loi touchant l'Hospice Cantonal. Voici quelques extraits de ce rapport, qui ne sont pas sans intérêt* :

«Le projet de loi que vous avez soumis à l'examen de la Commission dont j'ai l'honneur d'être l'organe est infiniment important. Il est aussi le plus digne de fixer les regards d'une Assemblée de législateurs républicains. Recueillir des malades que leur peu de moyens privent des secours nécessaires, - loger, soigner, alimenter des personnes affligées de maux incurables, - ouvrir un asyle aux infortunés qui ont perdu ce divin flambeau de la raison qui nous éclaire, et concilier à l'égard de tous ce qu'exige l'humanité et ce que commande la sûreté publique, tel est le noble but vers lequel vous tendez, Citoyens Législateurs. La patrie vous observe, l'indigence réclame votre pitié, tout le Canton attend avec une espérance mêlée d'inquiétude le moment si désiré où vous lui accorderez l'inestimable bienfait d'un Hospice Cantonal.

Profondément pénétrée de ces réflexions, jalouse de répondre à la confiance dont vous l'avez honorée, votre Commission n'a rien négligé pour donner à ce projet de loi cette maturité d'examen et cette somme de perfection qui peuvent seules le rendre digne d'être accepté par vous. En conséquence, après avoir approuvé la division proposée par le Petit-Conseil, savoir

$1^{\circ}$ d'un hôpital de malades à placer dans le cy-devant Hôpital de Lausanne

$2^{\circ}$ d'une maison des aliénés et d'un établissement d'incurables à placer dans le domaine appelé Champ-de-l'Air

elle s'est transportée dans ces deux emplacements et elle les a examinés.»

Après quelques passages sur l'hôpital général, le rapporteur poursuit: «Votre Commission s'est ensuite transportée au bâtiment du Champ-de-l'Air qu'elle a visité avec la même exactitude. Il serait à désirer sans doute que ce bâtiment fut plus régulier, qu'il offrit moins de place perdue, que la construction en fut plus

* Je remercie M. et $\mathrm{M}^{\mathrm{me}}$ Vallon de leur aide pour retrouver ces documents. 
solide. Néanmoins tel qu'il est actuellement, il répond aux vues du projet de loi. Un air pur, une situation agréable, des points de vue charmans, un mélange de prés, de vignes, de bois au-dessous desquels s'étend une ville populeuse, tout semble dire aux âmes sensibles que voilà le lieu où la patrie doit ouvrir un asyle à ses infortunés enfans.

On trouve dans ce bâtiment un emplacement susceptible de devenir avec peu de frais une maison d'incurables qui pourrait contenir je ne dis pas seulement les 30 lits que vous proposez pour ces sortes de malades, mais même 40 ou 50 si vous jugiez dans votre sagesse de devoir aller jusque-là.»

Ce rapport de Commission fut signé par Louis Bridel, professeur et président de la Commission, le 18 mai 1810.

Mais qu'était donc ce bâtiment du Champ-de-l'Air que la Commission a visité et où le Grand-Conseil a décidé, en 1810, de placer les malades mentaux ?

Chavannes dira dans ses notices sur l'Hospice Cantonal (1838): «C'était jadis une maison de campagne qui n'avait nullement été construite pour un établissement du genre de celui dont il s'agit. Son choix était la suite de circonstances auxquelles on a dû céder. On en a cependant tiré tout le parti possible.»

Avec la création d'un hôpital pour aliénés, séparé aussi bien de la médecine générale que de la prison, le jeune canton de Vaud a fait œuvre de pionnier. Si nous suivons Jetter dans son ouvrage extrêmement complet sur les hôpitaux psychiatriques du siècle passé, nous remarquons que rares sont les pays qui, en 1810, ont déjà créé des institutions spéciales pour malades mentaux. En Allemagne, nous pouvons citer Bracke près de Lemgo en 1811, Siegburg et Leubüs, Münster en 1814, etc.

Quant à l'organisation interne de cet Hospice des aliénés, comme il s'appelait encore en 1830, je n'en dirai pas grand'chose, car l'essentiel a été résumé dans le volume cité écrit par M. Secrétan. Quatre médecins se sont succédés au Champde-l'Air: d'abord Henri-Louis-Emmanuel Perey, qui cumula de 1811 à 1821 la fonction de médecin de l'Hôpital cantonal avec celle de médecin de l'Hospice des aliénés; ensuite de 1822 à 1833 Charles-Albert Perret qui a écrit un petit livre sur le Champ-de-l'Air; dès 1833 nous voyons à l'œuvre Charles Pellis jusqu'en 1845; le dernier fut Georges Zimmer.

Dans le livre de Perret, nous trouvons de nombreuses remarques intéressantes sur l'organisation interne de son Hospice, mais aussi sur les soins apportés aux malades, soins qui ne diffèrent guère de ceux décrits par les auteurs classiques de cette époque, à savoir Pinel, Esquirol, Jacobi, Roller, Benjamin Rush, etc. Une de ses principales préoccupations fut l'absence de séparation possible entre curables et incurables, principe qui s'est avéré plus tard extrêmement néfaste pour le développement de la psychiatrie. 


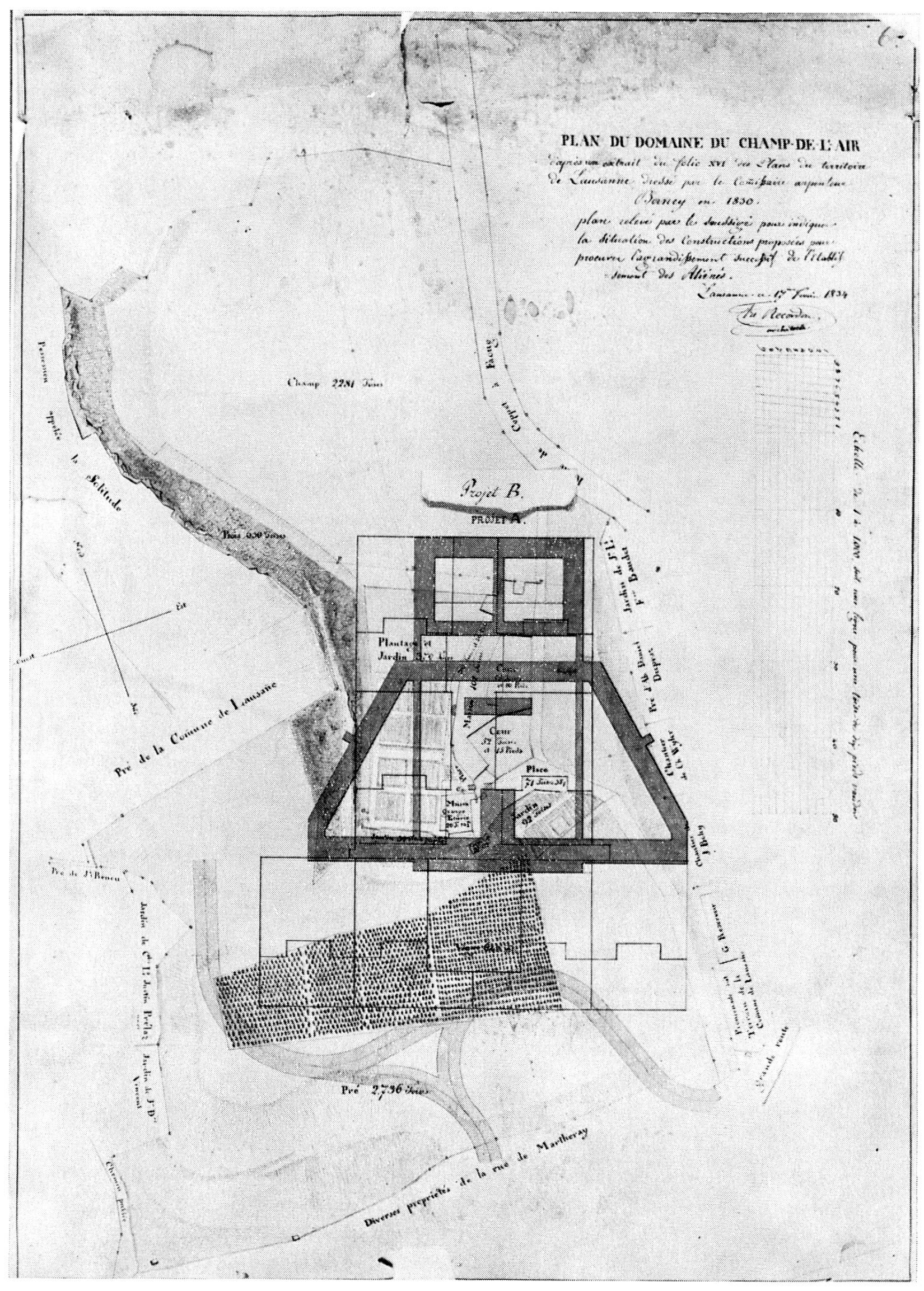


L'Hospice du Champ-de-l'Air a hébergé en 1838, d'après le rapport de Chavannes, une centaine de malades. Chavannes dit: «Pendant l'année 1837, le nombre de ceux qui y ont séjourné ou circulé a été de 140, 74 hommes et 66 femmes, dont une partie y ont passé plus ou moins de temps et l'autre, la plus considérable, se composait d'incurables qui forment un fond permanent.»

Il est intéressant de noter dans le livre de Perret que les malades bénéficiaient déjà d'un certain confort. Chacun avait son lit et son matelas. Les dortoirs ne comptaient pas plus de 6 lits. De même, on note un degré élevé d'occupations et de distractions pour les malades. Mais pourtant le bâtiment ne pouvait pas donner satisfaction à la longue et déjà à partir de 1835, les commissions du GrandConseil se préoccupent d'un remplacement éventuel du bâtiment du Champ-del'Air. En 1861, dans une observation adressée au Conseil d'Etat, on lit: «Chacun est d'accord que le Champ-de-l'Air est bien loin d'être à l'égal de ce qu'on est en droit d'exiger dans le canton de Vaud et qu'il y a urgence de faire cesser cette espèce de provisoire qui ne peut que jeter de la froideur chaque fois qu'il s'agit de réparation tant soit peu importante.»

La même année, l'Etat achète la propriété du Bois de Cery et c'est là où sera construite 1873 notre clinique, c'est-à-dire l'Hôpital de Cery. A-t-on songé à construire non pas à Cery, mais à l'emplacement du Champ-de-l'Air? Un vieux plan que j'ai découvert dans les archives de notre hôpital semble le prouver (voir figure). Ce plan nous montre en même temps les dispositions des bâtiments du Champ-de-l'Air.

1873 marque la fin de cet Hospice. Les malades sont transférés, mais le bâtiment connaît encore différentes destinations. Il reste propriété de l'Etat de Vaud, accueille très passagèrement les malades évacués du bâtiment de la Cité-Dessous voué désormais à l'enseignement secondaire. De 1884 à 1923 on donne au Champde-l'Air, d'après M. Secrétan, des cours d'agronomie et de zootechnie. On y plante un jardin botanique. En 1930 enfin, toujours selon M. Secrétan, le Champ-de-l'Air abrite le premier institut psychotechnique vaudois et c'est en 1954 qu'il est démoli.

Mais revenons à notre introduction et à la question de l'origine de cet Hospice. Euvre philanthropique ou lieu de réclusion pour les personnes improductives? Je ne peux m'empêcher de citer la fin de ce petit livre de Perret, publié en 1830. Il dit: «Il y a pour les familles, en particulier, et, dans notre pays, pour les communes aussi, un devoir sacré à remplir envers celui qui, bien loin de recouvrer sa raison, en reste privé à jamais. C'est ce respect de l'homme pour l'être qui porte face humaine, qui, dans quelque état qu'il soit, le garantit de l'abandon, du ridicule et du mépris. Puisse ce sentiment être assez généralement partagé pour améliorer le sort de quelqu'un de ces infortunés.» 
Rien ne laisse done supposer qu'à cette époque un esprit mercantile et froidement opportuniste aurait poussé les responsables à envisager la création d'un lieu de séjour pour des malades psychiquement atteints. Rappelons-nous aussi qu'en 1810, moment décisif pour la création du Champ-de-l'Air, le canton de Vaud était un canton agricole. Où donc aurait été ce fabricant décidé à vouloir se débarrasser de ses ouvriers non productifs? Non, je pense que l'exemple du canton de Vaud montre bien que les hypothèses de Foucault et de Dörner ne sont que partiellement justes. Quoi de plus naturel qu'un jeune Etat, fier de son autonomie, voulant le progrès, cherche à sortir ses malades mentaux de leur lieu d'hébergement insalubre et inconfortable!

L'Hospice d'aliénés du Champ-de-l'Air a d'ailleurs eu l'honneur de figurer dans la littérature mondiale. Dans une nouvelle d'August Strindberg « Gewissensqualen », nous trouvons le héros, officier de réserve allemand, qui devient psychotique au cours de la guerre franco-allemande et recouvre sa raison dans l'atmosphère du Champ-de-l'Air*.

* Je dois cette référence à M. Secrétan.

\section{Bibliographie}

Chavannes, D.-A., Notice sur l'Hospice du Canton de Vaud. (L'exemplaire de cette brochure que possède la Bibliothèque cantonale et universitaire porte la signature d'H. Druey.)

Commission du Grand Conseil, Rapport de la Commission nommée pour l'examen d'un projet de loi touchant l'Hospice Cantonal. Onglet cote K II 19/1810, Bibliothèque cantonale et universitaire, Lausanne.

Dörner, K., Psychiatrie und Gesellschaftstheorien. In: Psychiatrie der Gegenwart, Bd. I/1, 2.Aufl., Berlin, Springer, 1979, p1. 771-811.

Foucault, M., Folie et déraison. Histoire de la folie à l'âge classique. Paris, Plon, 1961.

Jetter, D., Geschichte des Hospitals. Westdeutschland von den Anfängen bis 1850. Bd.1, Wiesbaden, F.Steiner Verlag GmbH, 1966.

Perret, C., De l'Hospice des aliénés dans le canton de Vaud, Lausanne, Loertscher, 1830.

Secrétan, C., L’Hôpital de Cery 1873-1973, Lausanne, Payot, 1973.

Strindberg, A., Gewissenqualen. In: A.Strindberg, Werke VIII, München, A.Langen/ G.Müller, 1959, pp. 7-55.

\section{Summary}

The first psychiatric hospital in the canton of Vaud, in French speaking Switzerland, was Champ-de-l'Air. It was replaced in 1873 by the actual buildings of Cery near Lausanne.

Prof. Dr. med. Christian Müller

Hôpital de Cery

CH-1008 Prilly/Lausanne 